\title{
Development of Time Measuring Technique to Measure the Shock Speed during the Propagation in the Free Atmosphere
}

\author{
M. M. Ehsan ${ }^{1}$, R. B. Hossain ${ }^{2}$, S. Salehin ${ }^{3}$, M. A. Jinnah ${ }^{4}$ \\ Department of Mechanical and Chemical Engineering, \\ Islamic University of Technology (IUT), Dhaka, Bangladesh \\ E-mail: ${ }^{1}$ mme.ehsan@gmail.com, ${ }^{2}$ crawling_iut@yahoo.com, ${ }^{3}$ siin_salehin@yahoo.com, ${ }^{4}$ Jinnah@iut-dhaka.edu
}

\begin{abstract}
In the study of wave propagation in the free atmosphere, it is desirable to obtain time measurements to an accuracy of microsecond level. An experiment has been conducted to measure the wave speed during the propagation in the free atmosphere by the present technique. Due to sudden rupture of the diaphragm for the chamber pressure of $4.2 \mathrm{~kg} / \mathrm{cm}^{2}$ and a wave generating in a shock tube travels with subsonic speed and the measured incident wave Mach number is 0.8 which leaves the shock tube in the free atmosphere. The measured travelling time of the incident wave to travel $61.5 \mathrm{~cm}$ distance in the shock tube is $2200 \mu \mathrm{sec}$ and the same technique can apply to measure the shock wave speed. Several trigger points are installed at the exit of the shock tube in the open atmosphere to measure the strength of the wave propagation. It is observed that the wave strength decreases during the wave propagation in free atmosphere. Due to spherical expansion behind the wave, the pressure across the wave decreases. A numerical simulation is also conducted on supersonic shock wave to determine the shock speed and the travelling time in the free atmosphere. The pressures across shock wave at different locations of the shock wave are determined by solving the Euler equations and the simulation results indicate that the shock speed decreases during the propagation in the free atmosphere. In both experimental and numerical results, it is observed that the strength of the wave propagation in the free atmosphere decreases continuously due to spherical expansion. The present technique can also be used to measure the supersonic jet velocity, the velocity of bullet and any particle velocity in subsonic or supersonic ranges.
\end{abstract}

Keywords — Piezoelectric crystal; Subsonic and Supersonic wave; Free Atmosphere.

\section{INTRODUCTION}

The investigation of wave propagation in the free atmosphere is one of the complex experimental works. Experimental investigation is essential to improve the understanding of wave propagation with subsonic or supersonic speed in the free atmosphere. Some optical techniques can able to visualize the propagation and the location of the wave. High speed camera is also used for the flow visualization to analysis the structure of the wave. The application of some of these techniques to supersonic and hypersonic flows can be highly challenging due to the high velocity, strong gradients and restricted optical access generally encountered. Widely used qualitative and semiquantitative optical flow diagnostics are shadowgraph, schlieren, and interferometry. Schlieren visualizations of weak shock waves from common phenomena include loud trumpet notes, various impact phenomena that compress a bubble of air, bursting a toy balloon, popping a champagne cork, snapping a wooden stick, and snapping awet towel. The balloon burst, snapping a ruler on a table, and snapping the towel and a leather belt all produced readily visible shock wave phenomena.Doig et al 2008 conducted experiment where direction-indicating colour schlieren flow visualization was used to determine optically the general effectiveness of the methods. Multiple images were taken during a single tunnel run, which allowed, to some extent, confirmation of the general steadiness of the flow. The light source used in the experiments had a flash duration of $200 \mu \mathrm{s}$, which was short enough to 'freeze' any large-scale motions in the flow but long enough to average out small-scale fluctuations such as free-stream turbulence and boundary layer effects on the test section windows. Craig (1977) investigated efects of various area dis-continuities and perforations in a tube on the attenuation of weak shock waves. Analytical models of shock wave attenuation over perforated walls were based mostly on the formulation of one-dimensional unsteady compressible flow theory such as those developed by Szumowski (1971), and $\mathrm{Wu}$ and Ostrowski (1971). Sugimoto (1992) proposed the installation of an array of Helmholtz resonators in high-speed train tunnels. The design was based on nonlinear acoustics. The resonators were found to be effective for preventing the transition from acoustic waves to a shock wave by rendering them dispersive. However, once a shock wave was formed 
the device failed to counteract it. Onodera and Takayama (1990) experimentally determined a mass flow discharge coefficient through perforated walls by considering a twodimensional flow field. Takayama et al. (1995) installed a porous material on the wall of a real high-speed train tunnel and found that the porous wall indeed decreased the overpressure and smeared the pressure gradient of weak shock waves generated by high-speed trains. However, the level of sonic boom suppressions obtained in the laboratory was not attained in the real train tunnel.

Laser-based techniques such as laser Doppler anemometry and particle image velocimetry are well established for investigation of supersonic flows, but as yet their use in hypersonic flows has been limited. Other relevant measurement techniques include particle tracking velocimetry, Doppler global velocimetry, laser-two-focus anemometry, background oriented schlieren and laser induced fluorescence methods, molecular tagging velocimetry for velocity measurement and thermo-graphic phosphor thermometry for surface temperature measurement. Laser measurement techniques are becoming more commonly applied to many areas of thermo-fluids and heat transfer. An area of their application, which presents highly challenging requirements, is in the measurement of aerospace flows. In the shock wave research laboratory, Tohoku University Japan, the speed of projectile was measured by Shimadzu's HPV-1 High Speed Video Camera and the time elapsed from recording the first frame up to the $100^{\text {th }}$ frame was only about one ten-thousandth of a second. The ability to record dynamic images over short intervals is extremely significant. In the other technique, the projectile location can be determined by locating the trajectory of an incoming projectile. All supersonic projectiles are accompanied by a bow wave, often referred to as the "Mach Cone" which is virtually attached to the projectile and which can be detected acoustically. When a distributed array of microphones detect the arrival of this cone, it is possible to calculate the position and angle of the trajectory from the microphone data.

In the study of metal-high explosive detonation wave interaction, by the pin technique, it is desirable to obtain time measurements to an accuracy of less than 1 musec over a total time interval of $5 \mu \mathrm{sec}$. Commercial instruments capable of a high degree of time resolution were not available and a special instrument was developed for this purpose. A cathode-ray oscilloscope, displaying deflection type event pulses, is converted into a precise time-measuring instrument by a calibrated zigzag sweep. The record is obtained by single-shot photography of the cathode-ray tube sweep which is triggered by the event to be measured. Kobiera et al 2009 conducted experiment on the shock wave propagation through the test section and the shock wave causes a disturbance of the droplets; this process was visualized by a schlieren system and recorded by a high-speed camera. The camera was synchronized with the igniters that were connected to the spark plug mounted in the driver section. The test section was also equipped with a pressure measurement system (two pressure sensors and amplifiers) whose signals are recorded on an oscilloscope. The sensors were located ahead of the test section windows, and can be used to measure the shock velocity and pressure waveform. Pianthong et al 2002 conducted experiment with the use of the shadowgraph method, showing the projectile travelling inside and leaving the pressure relief section at a velocity of about $1100 \mathrm{~m} / \mathrm{s}$. they also visualized the supersonic diesel fuel jets (velocity about $2 \mathrm{~km} / \mathrm{s}$ ) by the shadowgraph method.

In the present method, metal to metal touch switches generate pulses for triggering which open and close the gate to allow and stop the crystal frequency for counting. Using the number of pulse counted, the travelling time between the trigger points is measured for the subsonic or supersonic objects. Due to pressure difference across the wave, the trigger is operated by the pressure response to detect the wave position in the open atmosphere. This is one of the easier methods to measure the travelling time of the subsonic or supersonic wave between the two trigger points as compare to other methods. Even visualizations of the wave front or the pressure response from pressure transducers are the familiar methods to measure the strength of the wave or its locations but the present technique is more reliable and accurate to measure the time. Normally the propagation time of the logic gates is not more than 20 nanoseconds, so the accuracy level of the measured time will be less than $0.006 \%$.

\section{EXPERIMENTAL SETUP}

The time measuring device has been constructed to measure the subsonic or supersonic wave propagation time in microsecond level in the free atmosphere. A piezoelectric crystal type HOSONIC, D349 which generates 3579540 pulses per second is used to measure the travelling time in micro-second level for any subsonic or supersonic objects in free atmosphere. A circuit has been developed by using two trigger points which is shown in Fig.1. Metal to metal touch switches generate pulses for triggering which open and close the gate to allow and stop the crystal frequency for counting.

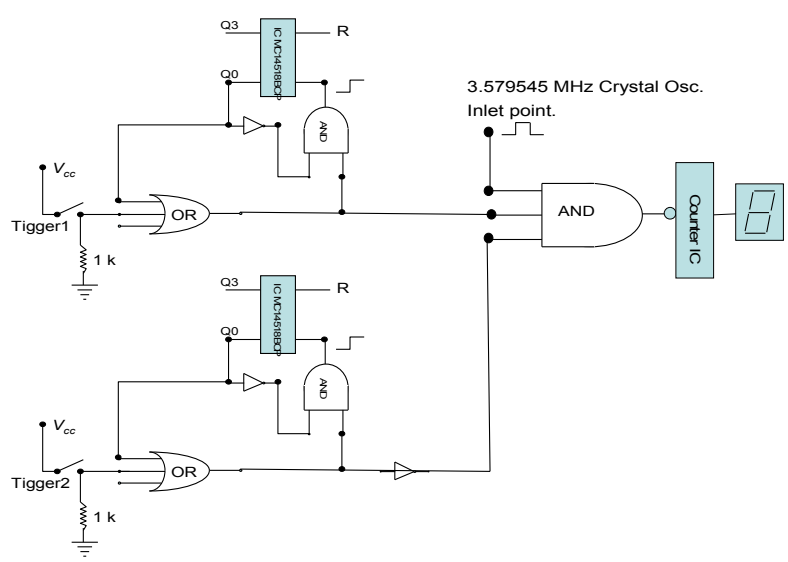

Fig 1 Circuit layout for time measuring device.

A direct metal to metal contact triggering is more efficient as compare to other sensing devices, like pressure transducers, Electro-optic sensors, Infrared sensors, Magnetic sensors etc. Using the number of pulse counted, the travelling time difference between the trigger points is measured for the subsonic or supersonic objects. To conduct an experiment on subsonic or supersonic objects, a shock tube is constructed in the Fluid Mechanics Laboratory of IUT for wave propagation in the free atmosphere. A compressor of $10 \mathrm{~kg} / \mathrm{cm}^{2}$ pressure range is used to raise the air pressure in the pressure chamber 
of the shock tube. The diameter and length of the pressure chamber is $15 \mathrm{~cm}$ and $1.5 \mathrm{~m}$. The dimensions of the driven section of the shock tube are $10 \mathrm{~cm}$ diameter and $5 \mathrm{~m}$ long. Due to sudden rupture of the diaphragm for the chamber pressure of range $3.5-4.5 \mathrm{~kg} / \mathrm{cm}^{2}$; a wave generates in a shock tube which travels with subsonic speed. If pressure range increases, a supersonic shock wave generates. Two trigger points (Trigger-1 and Trigger-2) are installed $61.5 \mathrm{~cm}$ apart in the shock tube to measure the incident wave Mach number. Similarly other 2-5 trigger points are also installed at different positions by the support, shown in Fig.2, in the free space to measure the wave speed during the propagation in the free atmosphere and the measured travelling times between different triggers are recorded.

\section{NUMERICAL METHODS}

The two-dimensional Euler equations are solved for the shock wave propagation in the free atmosphere. The governing equation is,

$$
\frac{\partial Q}{\partial t}+\frac{\partial F}{\partial x}+\frac{\partial G}{\partial y}=0
$$

Where $\mathrm{Q}$ is the vector of conservative variables, $\mathrm{t}$ is the time; $\mathrm{F}$ and $\mathrm{G}$ are the inviscid flux vectors. The governing equation described above for compressible inviscous flow is discretised by the finite volume method. A second order, upwind Godunov scheme of Flux vector splitting method is used to discrete the inviscid flux terms where HLL-Riemann solver is used for shock capturing in the flow. Two dimensional cells with adaptive grid systems are used for these computations. In this grid systems, the cell-edge data structures are arranged in such a way that each cell contains four faces which are sequence in one to four and each face indicates two neighbouring cell that is left cell and right cell providing all faces of a cell are vectorized by position and coordinate in the grid systems. The initial two-dimensional grid systems are shown in Fig.3.

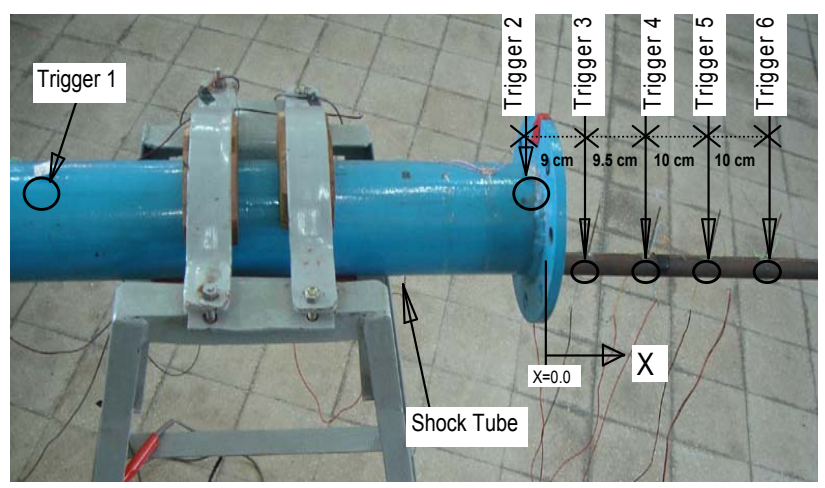

Fig 2 Shock tube and the position of triggers

The physical size of each cell before adaptation is equal to $13 \times 13(\mathrm{~mm})$. The grid adaptation is one of the improved and computational time saving techniques, which is used in these computations. The grid adaptation is performed by two procedures, one is refinement procedure and another is coarsening procedure. The refinement and coarsening operations are handled separately in computation. The upstream of incident shock wave is set as inflow boundary condition, the properties and velocities of which are calculated from Rankine-Hugoniot conditions with incident shock Mach number. The downstream inflow boundary conditions are shown in Fig.3 and the shock tube wall surface are used as solid boundary conditions where the gradients normal to the surface are taken zero.

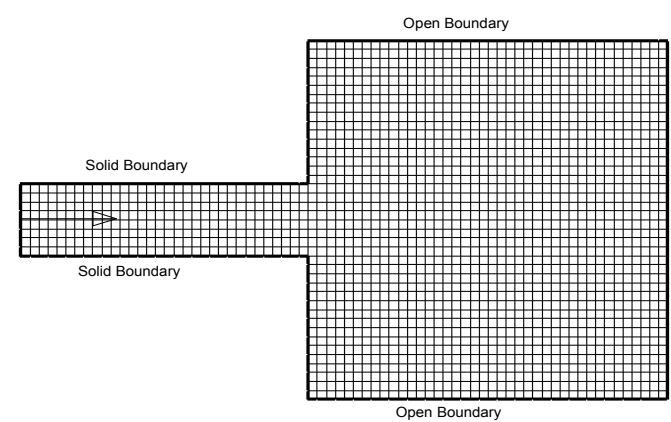

Fig 3 Numerical grids of the shock tube and the free space where solid boundaries are for tube and open boundaries are for free space.

\section{RESULTS AND DisCUSSION}

In the present works, the wave propagation in the free atmosphere has been studied in experimentally and numerically. An experiment has been conducted to measure the speed of the subsonic wave during propagation in the free atmosphere.

For the measurement of the wave speed at different positions in the free atmosphere, 5 trigger points are installed at the outlet of the shock tube which is shown in Fig.2. Each trigger point detects the pressure gradient of the wave front and finally estimate the travelling time of the wave propagation between the consequent trigger points in the free atmosphere. It is observed that the strength of the subsonic wave continuously decreases due to spherical expansion in the free atmosphere.

TABLE I

EXPERIMENTAL RESULTS

\begin{tabular}{|l|l|}
\hline Chamber pressure & $4.2 \mathrm{~kg} / \mathrm{cm}^{2}$ \\
\hline Mach number & 0.8 \\
\hline Travelling time & $2200 \mu \mathrm{sec}$ \\
\hline Number of triggers & 5 \\
\hline $\begin{array}{l}\text { Distance between the triggers in } \\
\text { the incident tube }\end{array}$ & $61.5 \mathrm{~cm}$ \\
\hline $\begin{array}{l}\text { Distance between the triggers in } \\
\text { the open atmosphere }\end{array}$ & $10 \mathrm{~cm}$ \\
\hline
\end{tabular}




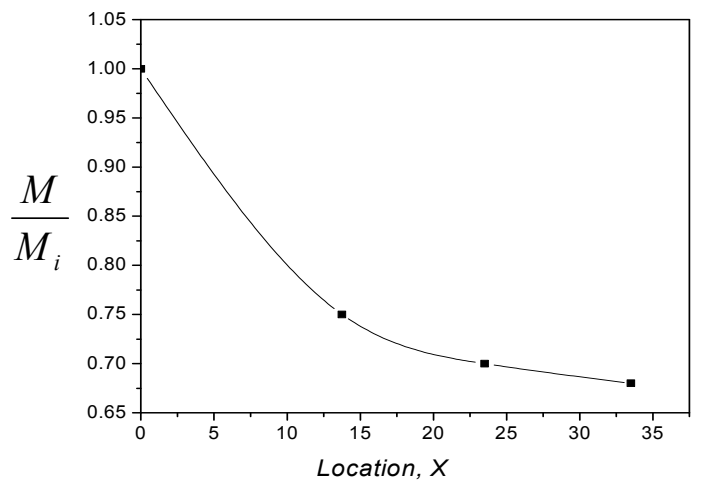

Fig 4 Experimental results of the subsonic wave speed between different triggers where the incident wave Mach, $M_{i}=0.8$.

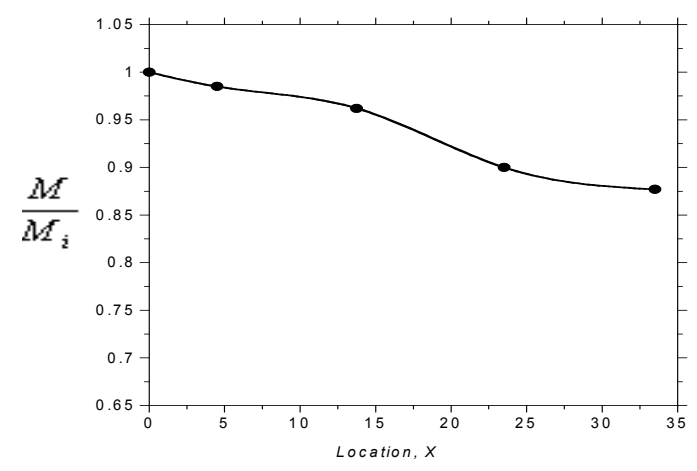

Fig 5 Numerical results of the supersonic wave speed between different triggers where the incident shock wave Mach, $M_{i}=1.3$.

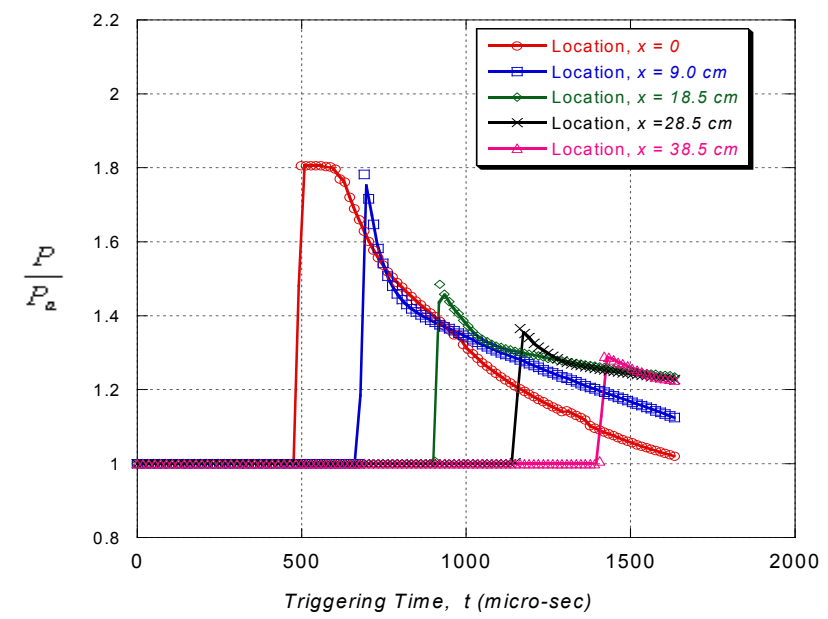

Figure 6: Numerical results of the pressure profiles at different trigger locations for the shock wave travelling in the free atmosphere.

For the incident subsonic wave Mach number, $M_{i}=0.8$, the decreasing wave Mach numbers, $M$ between the different trigger points are found which are shown in Fig.4. The present measurement technique to measure the wave speed is easier and more accurate as compare to other techniques. Visualizations of the wave by photographic techniques are also very familiar methods but optical setup and locating the wave position are the complex matter. Using pressure transducers by measuring the pressure response at different location of the shock tube, it is only possible to measure the incident wave speed inside the tube. Such type of technique can also apply to measure the supersonic shock wave speed at the outlet of the shock tube.

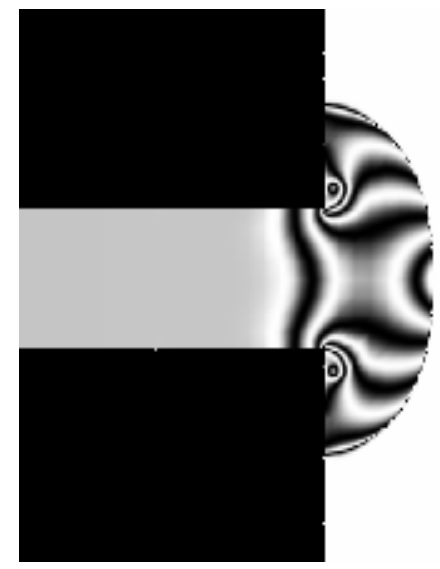

(a). $x=9.0 \mathrm{~mm}$ and density fringe step 0.2 .

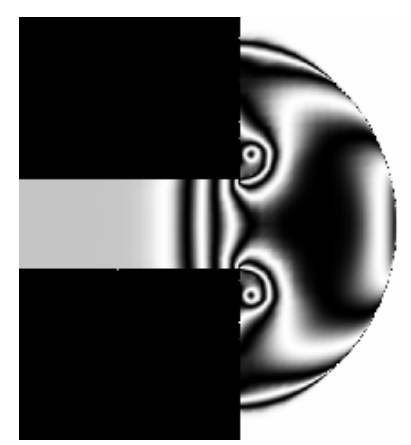

(b). $x=18.5 \mathrm{~mm}$ and density fringe step 0.2 .

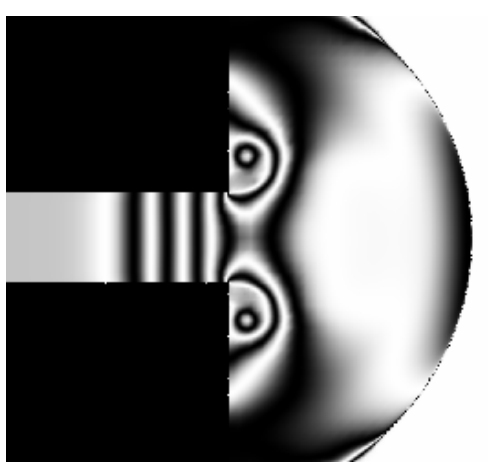

(c). $x=28.5 \mathrm{~mm}$ and density fringe step 0.2 .

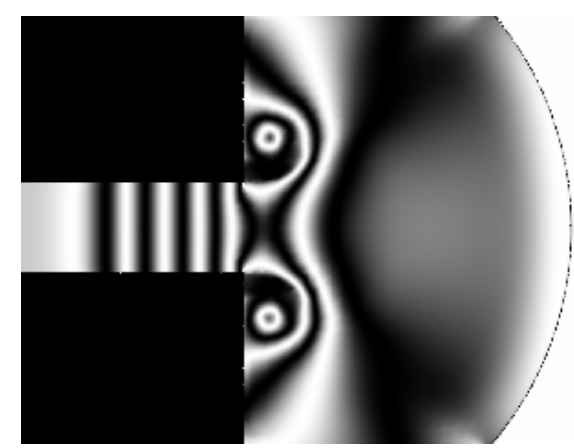

(d). $x=38.5 \mathrm{~mm}$ and density fringe step 0.2 .

Fig7 Numerical results of the density fringes at different locations of the shock wave in the free atmosphere. 
Numerical results of the supersonic shock wave propagation in the free atmosphere are also observed by solving the 2D Euler equations by grid adaptation technique. It is observed for the incident shock Mach number, $\quad M_{i}=$ 1.3 that the shock wave strength gradually decreases during the propagation in the free atmosphere which is shown in Fig.5.

The numerical results of the pressure history at different trigger points are shown in Fig.6. The pressures in this figure are normalized by the atmospheric pressure $(\mathrm{Pa})$. It is apparent from Fig.6 that at each point, the pressure throughout the investigated time will be lower after the shock wave travelling over the trigger points. As a matter of fact, the spherical expansion of the wave gradually decreases the wave strength, as well as the pressure across the wave. The pressure history at the outlet point that is trigger point 2; where $\mathrm{x}=0.0 \mathrm{~mm}$, is shown in Fig. 6 and it is observed that the pressure throughout the investigated time decreases sharply due to faster expansion wave travelling towards the shock tube outlet face from the opening space.

Numerical results of the density fringes for the shock wave position at each trigger point are observed to analysis the expansion wave propagation and front shape. It is observed in Fig.7 (a)-(d) that the shape of wave front changes gradually to becoming spherical and the expansion wave moving towards the centre of the spherical front and finally the expansion wave movement is observed in the shock tube.

\section{CONCLUSIONS}

In this paper, the wave propagation in the free atmosphere has been studied in experimentally and numerically. It is observed that the present wave speed technique is more suitable and comparatively easy. The metal to metal triggering techniques used in the present experiment are faster and more sensitive as compare to other triggering techniques and it is possible to measure both the subsonic and supersonic wave speed by this method. High pressure gradient across the wave front can able to detect the wave position in the free atmosphere and using consequent several trigger points, the decreasing wave strength is observed far from the shock tube outlet. Shock tube is used in the present experiment to generate subsonic wave in the free atmosphere. Due to insufficient facilities in the Laboratory, experiments on supersonic shock wave are avoided. Numerical computations on supersonic shock wave are conducted and it observed that the wave decreases gradually after the wave propagation in the free atmosphere from the outlet of the shock tube face. The numerical results of the density fringes indicate the expansion wave propagation towards the shock tube outlet and it is observed that wave front is spherical in shape.

\section{REFERENCES}

[1] Doig, G.C., Barber, T.J., Leonardi, E., Neely, J., Kleine, H., 2008 Methods for investigating supersonic ground effect in a blowdown wind tunnel, Shock Waves, 18: 155-15.

[2] Kobiera, A., Szymczyk, J., Wola'nski, P., Kuhl, A., 2009. Study of the shock-induced acceleration of hexane droplets, Shock Waves, 18 475-85.

[3] Pianthong, K., Zakrzewski, S., Behnia, M., Milton, B.E., 2002. Supersonic liquid jets: Their generation and shock wave characteristics, Shock Waves, 11: 457-66.

[4] Craig JE (1977) Shock waves in open ended ducts with complex geometry.Ph D Aeronautics. California Institute of Technology,Pasdene CA,USA.

[5] Szumowski AP (1971) Attenuation of Shockwaves along a perforated tube. In: Stollery JL, Gaydon AG, Wen PR (eds) Shock Tube Research, Proc 8th Int Symp Shock Tubes. Chapman \& Hall 14.

[6] Sugimoto N (1992) Propagation of nonlinear acoustic waves in a tunnel with an array of Helmholtz resonators. J Fluid Mech 244: 55-78.

[7] Onodera H, Takayama K (1992) An analysis of shock wave propagation over perforated wall and its discharge coefficient. Trans Jpn Soc Mech Eng B (in Japanese). 58:1408.

[8] Takayama K, Sasoh A, Onodera O, Kaneko R, Matsui Y (1995) Experimental investigation on tunnel sonic boom. Shock Waves $5: 127\{138$ 